\title{
Effects of Water-Based Contrast Media on Experimentally Induced Established Atherosclerotic Plaques in Rabbits
}

\author{
Kaur M. ${ }^{a, b^{*}}$, Rahman $T^{b}$, Mohd Kornain $N K^{b}$, Ahmad $R^{b}$, Awang Jalil $R^{b}$, Isa $M R^{b}$, Mohd Noor $N^{b}$, Nawawi $H^{a, b}$ \\ anstitute for Pathology, Laboratory and Forensic Medicine (I-PPerForM), Universiti Teknologi MARA Sungai \\ Buloh, Selangor, Malaysia \\ ${ }^{\mathrm{b}}$ Faculty of Medicine, Universiti Teknologi MARA Sungai Buloh, Selangor, Malaysia
}

\section{ABSTRACT}

INTRODUCTION: Imaging techniques involving optical coherence tomography, computed tomography (CT) and high-resolution magnetic resonance imaging (MRI) are used as tools to identify atherosclerotic plaques. However, the effects of water-based contrast media used in Post Mortem Computed Tomography Angiography (PMCTA) on the histopathology of atherosclerotic plaques have not been widely explored. The objective of this study is to determine the effects of water-based contrast media used in PMCTA on the histopathology of atherosclerotic plaques and biomarkers of atherosclerosis in experimentally induced established atherosclerotic rabbits. MATERIALS AND METHODS: Twenty male New Zealand white rabbits were divided into 2 groups. One group was given a high cholesterol diet (HCD) for 12 weeks to establish atherosclerosis and the control group normal diet (ND). Five rabbits from each group were then given intravenous water-based contrast media before being sacrificed. The entire length of aorta was dissected and submitted for histopathological examination and determination of tissue biomarkers a-SMA and MMP-9. RESULTS: Histopathological examination of the aorta including percentage of area covered by plaque and foam cell formation showed no significant difference in atheromatous plaque formation in both groups of $\mathrm{HCD}$ rabbits with or without intravenous contrast media injection (plaque: $55 \pm 41$ vs. $63 \pm 15, p=0.731$; foam cells: $124 \pm 83$ vs. $171 \pm 55, p=0.325$ ). Similarly, $a-S M A$ and MMP-9 protein expression also showed no significant difference in both groups ( $\mathrm{a}-\mathrm{SMA}: 70 \pm 20$ vs. $67 \pm 26, \mathrm{p}=0.807$; MMP-9: $60 \pm 12$ vs. $57 \pm 17, \mathrm{p}=0.785$ ). CONCLUSION: Water-based contrast media used in PMCTA does not affect the morphology or the immunohistochemistry staining of SMA and MMP-9 in atherosclerotic plaques.

KEYWORDS: atherosclerotic plaques, biomarkers, histopathology, post mortem computed tomography angiography and water- based contrast media

\section{INTRODUCTION}

Cardiovascular disease which includes coronary artery disease (CAD) and stroke are the most common non communicable disease globally accounting for 17.8 million deaths in $2017 .{ }^{1}$ In Malaysia, non- communicable diseases accounted for $74 \%$ of all deaths of which $35 \%$ were due to

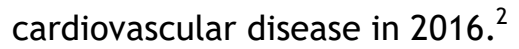

Corresponding Author:

Dr Mansharan Kaur Chainchel Singh

Institut Patologi, Perubatan Makmal dan Forensik (I-PPerForM), Aras 4,

Bangunan Akademik, UiTM Kampus Sungai Buloh, Jalan Hospital, 47000 Sungai

Buloh, Selangor, Malaysia.

Tel No: +603- 61267466,

Email: mansharan70@gmail.com,

mansharan@salam.uitm.edu.my
Autopsy is considered the reference standard for post-mortem evaluation regarding the cause of death. In most countries, autopsies are performed with permission from family members of the deceased. Due to the invasive nature of autopsies, permission to conduct autopsy is often met with initial resistance by family members. Furthermore, autopsies are time consuming and labour intensive. The current declining number of autopsies could mean that valuable data regarding actual traumatic injuries and causes of death will be either difficult to obtain, misclassified, or even lost. . $^{3-8}$ To overcome this problem, several studies have been conducted to determine the value of post-mortem imaging as an alternative to clinical autopsy.

The use of computed tomography (CT) was first reported in association with post-mortem practice 
in 1983. ${ }^{9}$ Since then, the use of multi-detector computed tomography (MDCT) and magnetic resonance imaging have been widely reported within forensic and radiological literature. ${ }^{3-8,10-14}$ Although post mortem computed tomography (PMCT) represents the best way to visualize the skeletal system, this technique is not suited for vascular diagnosis.

Therefore, the application of contrast media as used in clinical radiology is a promising next step which mirrors clinical practice, in which $\mathrm{CT}$-angiography (CTA) is an excellent method for assessing acute and chronic coronary artery disease as it allows investigation of the arterial lumen and vascular wall diseases. The use of contrast in forensic setting i.e. post-mortem computed tomography angiography (PMCTA) has been shown to aid and assist the pathologist in identifying specific sites of vascular pathology for targeted postmortem analysis.

However, the implementation of cardiac MDCT in post-mortem setting is impaired by the lack of an active circulation to deliver suitable contrast agents via an intravenous route for which a modified heart and lung bypass machine or embalming machine may be used. ${ }^{15}$ Contrast agents used may include Angiofil (an oil-based contrast material) mixed with paraffin oil or aqueous solution, a mixture of polyethylene glycol and ultravist. ${ }^{16}$ Studies revealed that use of both these contrast agents did not produce differences in diagnostic significance on toxicology or biochemistry of blood, vitreous and urine samples. ${ }^{17}$, ${ }^{18}$ However, data looking at the effect of contrast media on the histopathology of atherosclerotic plaques and the immunohistochemistry staining markers reflecting atherogenesis remains scarce.

This is important to ensure that contrast media used in PMCTA does not affect the histopathology quality of the tissues of interest. The purpose of this study is to investigate if PMCTA using an aqueous solution mixture of polyethylene glycol and ultravist has any effect on the histopathology of atherosclerotic plaques and immunohistochemistry staining for tissue markers of atherogenesis using rabbit as an animal model. Although primates and pigs may serve as ideal animal models of vulnerable plaques, the high expense, restricted availability and ethical issues have limited their use in this field making rabbits by far the most useful large-animal models to study atherosclerotic plaques. ${ }^{19,20}$

\section{MATERIALS AND METHODS}

\section{Animal Care and Treatment}

Twenty adult male New Zealand white rabbits, Oryctolaguscuniculus were used in this animal experiment. The rabbits were obtained from Chenur supplier and housed at the Laboratory Animal Care Unit (LACU), University of Teknologi MARA (UiTM), Sungai Buloh. All rabbits were handled in accordance with the standard guide for care and use of laboratory animals. Ethical approval from the Institutional Animal Care and Use Ethics Committee [ACUC-6/14] was obtained prior to the commencement of this study. After a quarantine period of 2 weeks, the rabbits were randomly divided into two groups, control group fed with $100 \mathrm{gm}$ of standard chow diet per day (ND, $n=10)$ and high cholesterol diet group fed with $100 \mathrm{gm}$ of high cholesterol diet (HCD) composed of SF00-221 plus 1\% cholesterol per day $(\mathrm{HCD}, \mathrm{n}=10)$. The diets were administered for 12 weeks continuously to ensure the development of established atherosclerosis in the HCD group, defined by the presence of macroscopic atherosclerotic plaques. This study design was adopted from a previously published animal study using the same model. ${ }^{21}$ All animals were kept under a circadian rhythm of $12 \mathrm{~h}$ light: $12 \mathrm{~h}$ dark with free access to drinking water and housed in cages (1 rabbit per cage) with a floor area of $42 \mathrm{~cm}^{2}$ and a height of $45 \mathrm{~cm}$, including a raised area of approximately $55 \times 30 \mathrm{~cm}$.

At the end of 12 weeks, the rabbits sedated with a combination of ketamine, xylazine and water in the ratio of 5:3:2 at $1 \mathrm{ml} / \mathrm{kg}$ body weight delivered via intramuscular injection into the gluteal muscle. A total of five rabbits from each group were randomly selected and a 24G cannula was inserted percutaneously into the central auricular artery, flushed with heparin saline and fixed to the skin. The cannula was then connected to an IV drip containing a mixture of polyethylene glycol and ultravist $(1.5 \mathrm{mls} / \mathrm{kg}$ body weight) in the ratio of $10: 1$ using a drip rate of $5 \mathrm{drops} / \mathrm{min}$. At the end of the experiment the rabbits were euthanized and the aorta was then isolated for histopathology examination.

\section{Sudan IV staining}

The aortas were gently rinsed with normal saline, and cut and open longitudinally to expose the entire 
lumen and pinned flat on a polystyrene board. The opened aortas were fixed with $10 \%$ neutral buffered formalin (Richard Allan Scientific, USA) overnight. Then, the aortas were rinsed with $70 \%$ ethanol, followed by immersion with Herskhemers solution ( 5 $\mathrm{g}$ of Sudan IV dissolved in $500 \mathrm{ml}$ 70\% ethanol and 500 $\mathrm{ml}$ acetone) for another 15 minutes at room temperature. Thereafter the aortas were rinsed in running tap water for an hour to destain them. The stained aortas were put on a smooth contrast background for imaging and the entire aorta was captured using a digital camera.

\section{Sample Processing}

The entire length of aorta was divided into three: (1) aortic arch, (2) thoracic aorta and (3) abdominal aorta. The aortas were then fixed in $10 \%$ formalin solution and underwent overnight automated tissue processing procedure using a MICROM Automated Tissue Processor. The processed aortic tissues were embedded in paraffin wax, sectioned at $3 \mu \mathrm{m}$ thick tissue sections using a microtome, placed on coated slides, deparaffinised and hydrated gradually.

\section{Histological Examination}

The tissue sections were stained with haematoxylin and eosin (H\&E). The nuclei were stained with haematoxylin, rinsed with clean water and differentiated with $0.3 \%$ acid alcohol. The slides were then rinsed again, stained with eosin for 2 minutes and dehydrated before mounting. The histopathological examination was performed by 2 board certified histopathologists which were blinded and the findings of atheromatous plaque formation was quantified using a scoring system that ranged from absent to severe lesions (0: none, 1: mild, 2: moderate, 3: severe).

\section{Immunohistochemistry staining}

Endogenous peroxidise activity was blocked using 3\% hydrogen peroxide in methanol for 15 minutes at room temperature. The slides were then rinsed prior to antigen retrieval via microwave, and incubated for 20 minutes with a blocking serum at room temperature. Rabbit polyclonal antibody for a-SMA (Alpha-Smooth Muscle Actin) and MMP-9 (Matrix Metallopeptidase 9, Santa Cruz Biotechnology, USA) were added and incubated for an hour at room temperature. Next, biotinylated secondary antibody (Santa Cruz Biotechnology, USA) was added for 30 minutes, followed by peroxidase-conjugated streptavidin for another 30 minutes at room temperature. Finally, the slides were incubated with diaminobenzidine for 10 minutes in the dark to visualize the peroxidase production. a-SMA and MMP-

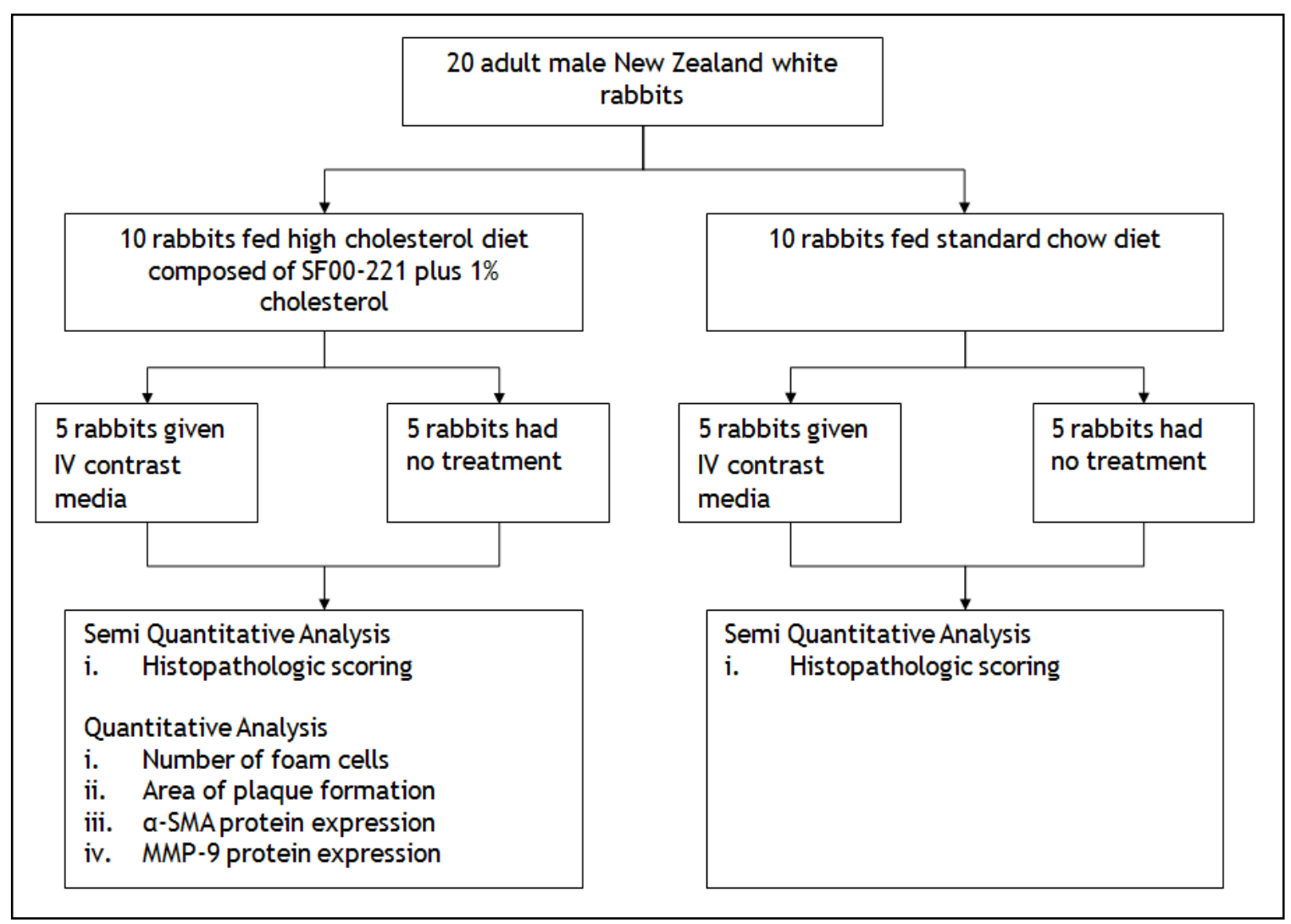

Figure 1: Flow chart of experiment 
9 protein expression were determined by endothelial cell staining positive (brown) to total lesion area and quantified using an image analyser software (analySIS ${ }^{\circledR}$ FIVE, Olympus, USA). The flow chart of the experiment is shown in Figure 1.

\section{Sample size determination}

The sample size of this study was determined using the crude method based on the law of diminishing return (resource equation). In this experiment, we decided to have 4 group of comparison (two arms, normal diet and high cholesterol diet. Each arm was then further subdivided into two groups, contrast media treatment and non- treatment) and to put 5 rats in each group to see the effect of different group. The sample size was calculated using the following formula by Charan and Kantharia: ${ }^{22}$

$E=$ (Number of animals per group $x$ number of groups) - Total number of groups

$$
E=(5 \times 4)-4
$$$$
E=16
$$

Since $\mathrm{E}$ is more than 10 , it can be considered that the sample size is adequate to detect a significance different between groups.

\section{Statistical analysis}

The statistical analysis package for social sciences (SPSS) for Windows, version 20.0 (Chicago, IL, USA) was used for all analyses. Histopathologic scoring of plaque formation in the aorta of rabbits fed with high cholesterol and normal diet with and without exposure to contrast media were tested using twoway ANOVA to validate the area of plaque formation. The comparison of mean percentage of area covered by atherosclerotic plaque, number of foam cells, aSMA and MMP-9 protein expression were measured only in the HCD group using independent T-Test. The level of statistical significance was considered at $\mathrm{P}<$ 0.05 .

\section{RESULTS}

Effects of contrast media on histopathologic scoring of plaque formation

Plaque formation in the HCD group occurred throughout the entire length of the aorta which included the aortic arch, thoracic aorta and abdominal aorta, while there was no evidence of plaque formation in the NC group with similar cell morphology in rabbits with or without intravenous contrast media injection [Figure 2 (a)(i), (a)(ii), (a) (iii) and (a)(iv)]. A two-way analysis of variance was used to test the histopathologic scoring of plaque in the aorta of HCD and NC rabbits with and without exposure to contrast media [Table I].

Table I: Histopathologic scoring of plaque formation in the aorta of rabbits on high cholesterol and normal diet with and without exposure to contrast media.

\begin{tabular}{|c|c|c|c|c|}
\hline Diet & Treatment & $\begin{array}{c}n \\
\text { value }\end{array}$ & $\begin{array}{c}{ }^{a} \text { Mean } \\
\pm S D\end{array}$ & $\begin{array}{c}{ }^{b} P \\
\text { value }\end{array}$ \\
\hline \multirow[t]{2}{*}{$\begin{array}{l}\text { High } \\
\text { Cholesterol }\end{array}$} & $\begin{array}{l}\text { No } \\
\text { treatment }\end{array}$ & 5 & $\begin{array}{l}1.90 \pm \\
0.43\end{array}$ & \multirow{2}{*}{${ }^{\mathrm{c}} 0.000^{* *}$} \\
\hline & $\begin{array}{l}\text { Contrast } \\
\text { media }\end{array}$ & 5 & $\begin{array}{l}1.90 \pm \\
0.89\end{array}$ & \\
\hline \multirow[t]{2}{*}{ Normal } & $\begin{array}{l}\text { No } \\
\text { treatment }\end{array}$ & 5 & $\begin{array}{l}0.00 \pm \\
0.00\end{array}$ & ${ }^{\mathrm{d}} \mathrm{NS}$ \\
\hline & $\begin{array}{l}\text { Contrast } \\
\text { media }\end{array}$ & 5 & $\begin{array}{l}0.00 \pm \\
0.00\end{array}$ & ${ }^{\mathrm{e}} \mathrm{NS}$ \\
\hline
\end{tabular}

Note: ${ }^{a}$ Data expressed as mean \pm SD. ${ }^{b}$ Analysed using twoway ANOVA. 'Between diet subjects. ${ }^{\mathrm{d} B e t w e e n}$ treatment

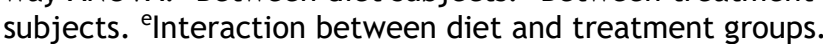
NS: Non significant. ${ }^{*} p<0.05 .{ }^{* *} p<0.01 .{ }^{* * *} p<0.001$.

$\mathrm{HCD}$ rabbits showed a higher histopathologic scoring $(F(1,16)=72.991, p=0.000, \eta 2=0.820)$ compared to $N C$ rabbits. $\mathrm{HCD}$ rabbits with and without contrast media treatment showed no significant difference $(F(1,16)$ $=0.000, \mathrm{p}=0.996, \mathrm{n} 2=0.000$ ). The interaction between diet and treatment group was not significant $(p=0.996)$ [Table II].

A comparison of the area covered by atherosclerotic plaque in the HCD group of rabbits did not show any significant difference between the group with and without exposure to contrast media $(55 \pm 41$ vs. $63 \pm 15$, $\mathrm{p}=0.731)$ [Figure $2(\mathrm{~b})(\mathrm{i})]$.

Table II: Summary of p-values from ANOVA for measured variables from normal diet and high cholesterol diet to contrast media treatment and non treatment.

\begin{tabular}{lccccc}
\hline Source & $d f$ & $M S$ & $F$ & $p$ & $\begin{array}{c}\text { Effect } \\
\text { size }\end{array}$ \\
\hline Diet & 1 & 18.031 & 72.991 & $0.000^{* * *}$ & 0.82 \\
Treatment & 1 & $5.00 \mathrm{E}-06$ & 0 & 0.996 & 0 \\
$\begin{array}{lccc}\text { Diet } \mathrm{X} \\
\text { Treatment }\end{array}$ & 1 & $5.00 \mathrm{E}-06$ & 0 & 0.996 & 0 \\
Error & 16 & 0.247 & & &
\end{tabular}

Note: $M S=$ Mean squares, effect size $=\eta 2 .{ }^{*} p<0.05 .{ }^{* *} p<$ $0.01 .^{* * *} p<0.001$ 


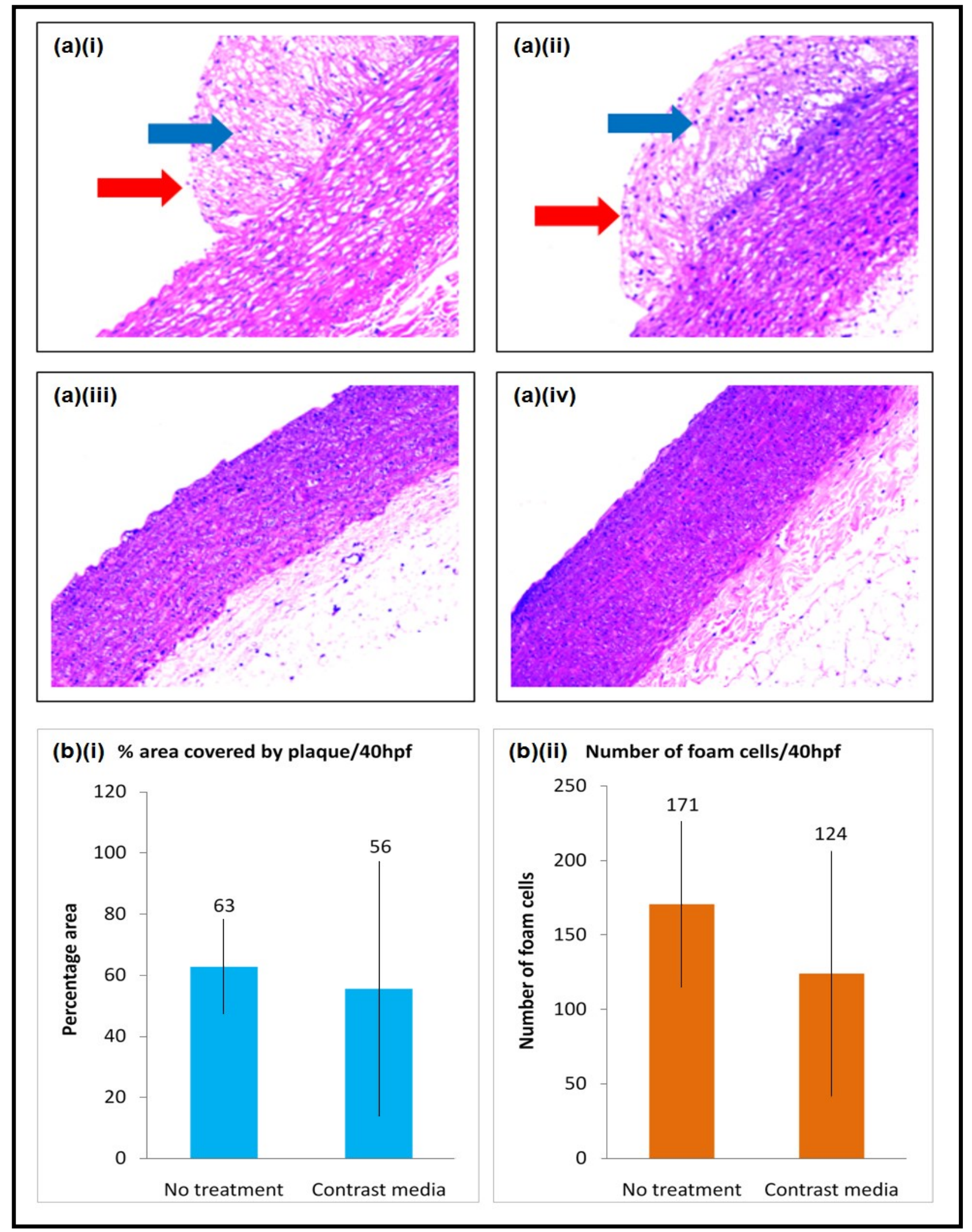

Figure 2: Histopathological examination of plaque formation in the aorta of HCD rabbits and NC rabbits with and without exposure to contrast media.

(a) Hematoxylin and eosin (H\&E) stain shows no significant difference in histopathological characteristics of atherosclerotic plaques (red arrows) and foam cell rich lesions (blue arrows) between both groups of HCD with and without contrast media (a)(i) and (a)(ii). No evidence of atherosclerotic plaque formation in both groups of NC with and without contrast media (a)(iii) and (a)(iv).

(b) Percentage area covered by plaque (b)(i) and number of foam cells (b)(ii). Data expressed as mean $\pm S D$. Analysed using independent T-Test. $n=5$. (a) Percentage of area covered by plaques under $40 x$ hpf. $p=0.731$ (b) Number of foam cells formation under 40x hpf. $p=0.325$. 
Effect of contrast media on a-SMA and MMP-9 protein expression

Tissue biomarkers were determined via IHC assay and neutral effects were observed on the protein expression of both $a-S M A(70 \pm 20$ vs. $67 \pm 26, p=0.807)$ and MMP-9 $(60 \pm 12$ vs. $57 \pm 17, p=0.785)$ of the HCD rabbit tissues in both contrast media and noncontrast media groups [ Figure 3 and 4]

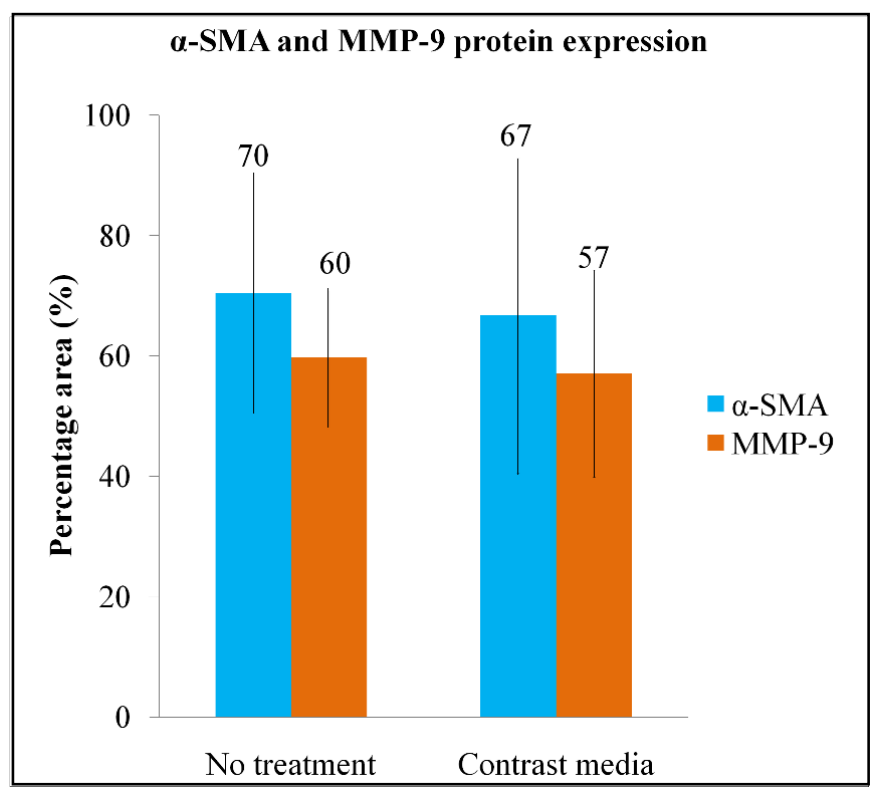

Figure 3. Immunohistochemistry (IHC) expression of aSMA and MMP-9 in contrast media and non-contrast media groups of HCD rabbits.

Data expressed as mean \pm SD. Analysed using independent $T$ Test. $\mathrm{n}=5$. $\mathrm{a}-\mathrm{SMA} \mathrm{p}=0.807$. MMP $-9 \mathrm{p}=0.785$.

Effect of contrast media on histopathological characteristics

Hematoxylin and eosin (H\&E) stain showed no significant difference in histopathological characteristics of atherosclerotic plaques between both groups of HCD with and without contrast media [Figure 2 (a)(i) and a(iii)].

\section{DISCUSSION}

The classic post-mortem examination is an intrinsically destructive process, potentially destroying subtle findings and leaving the corpse in a state unsuitable for a second autopsy. ${ }^{23}$ Furthermore, apart from objections from the next of kin and religious groups, the main problem is reproducibility in court. ${ }^{24}$ To overcome these problems, PMCT was introduced with the application of contrast media as used in clinical radiology being a promising next step which mirrors clinical practice, in which CTangiography (CTA) is the most widely used method
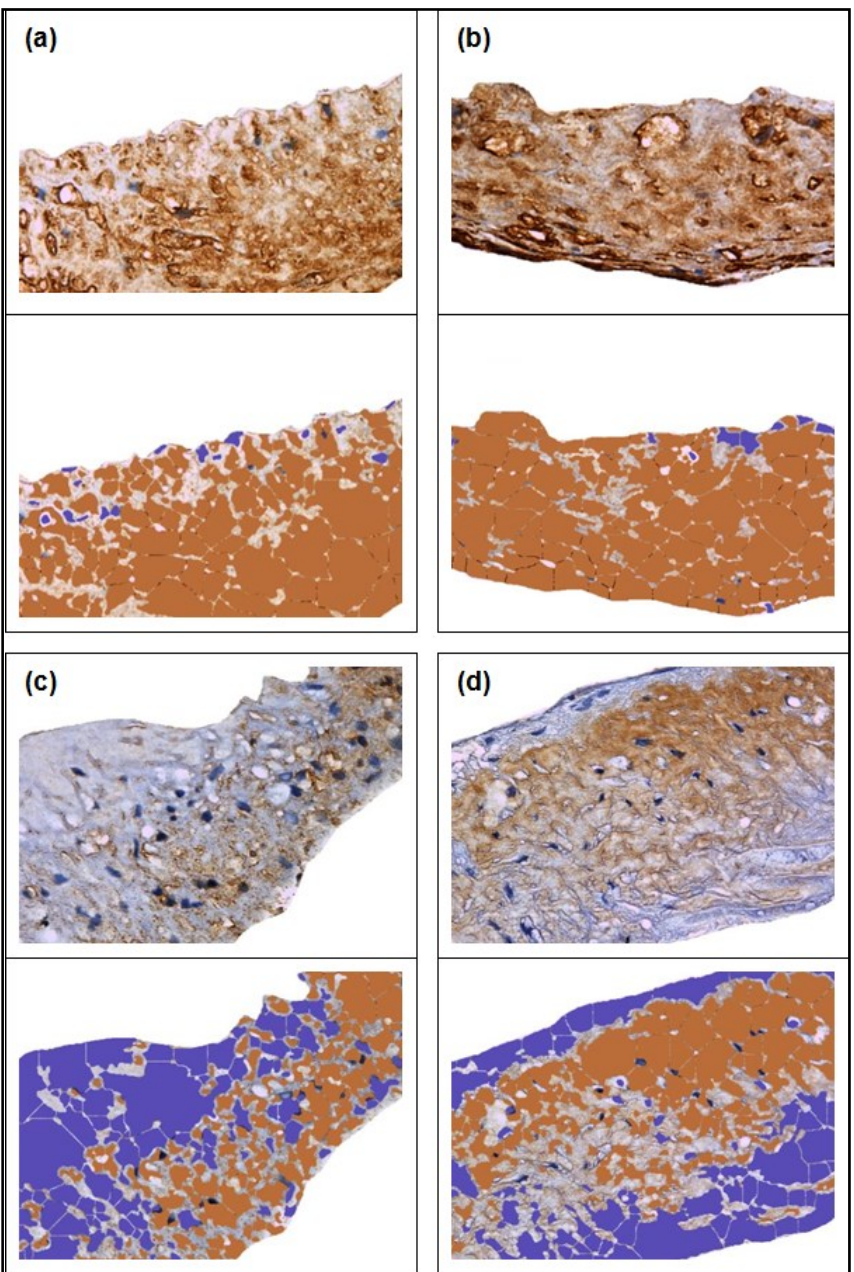

Figure 4. Cross section of immunohistochemistry (IHC) stain in the aorta of HCD rabbits and NC rabbits with and without exposure to contrast media.

IHC stain shows no significant difference in morphology of immunohistochemical stains for aSMA ( $a$ and b) and MMP-9 (c and d) between both groups of HCD and NC with and without contrast media.

for the detection and localization of vascular pathology. ${ }^{25}$

There have been a few studies with small numbers of human and animal corpses or organs that have addressed the possible use of water-based contrast media i.e. polyethylene glycol (PEG). Different mixtures of PEG were used in porcine hearts and in human corpses. ${ }^{25-27}$ The studies reported tissue enhancement properties possibly increasing diagnostic value of the method, while higher viscosity PEG solutions seemed to limit extravasation and over enhancement of various tissues. However, there are still unresolved concerns regarding the histological and biochemical alterations by contrast media in lipid-containing tissues as PEG acts as a solvent for lipids.

This was a pilot study determining the effects of water-based contrast on the morphology of induced atherosclerotic plaques and the 
immunohistochemistry staining of tissue markers of atherogenesis, namely SMA and MMP-9. Although there are currently three different contrast agents used in PMCTA, which are oil-based, water-based and air, we chose water-based contrast media (a combination of polyethlene glycol 200 and ultravist) as it is cheaper compared to the oil-based contrast media and can be used for whole body angiogram rather than targeted angiogram where air is limited to.

This study showed that water-based contrast media used did not have any effect on atherosclerotic plaques as evidenced by the fact that both the morphology of foam cells and plaques in the aortas of the rabbits fed with HCD did not show any changes whether exposed to contrast media or not. Furthermore, water-based contrast media used in this study also did not have any effect on the determination of tissue biomarkers of a-SMA and MMP -9. Expression of MMP-9 is associated with unstable plaque where MMP-9 concentration exhibits a dose dependent relationship with carotid plaque stability. MMP-9 also regulates both smooth muscle cells migration and proliferation in the development of arterial lesions. ${ }^{27,28}$ a-SMA is expressed at an early differential stage during smooth muscle differentiation and is observed especially on the luminal surface of atheromatous plaques. ${ }^{24}$

To the best of our knowledge, there has not been any previous study to determine the effects of contrast media used in PMCTA, be it water-based or oil-based, on coronary artery tissue morphology. These findings collectively suggest that future research to assess the reliability of PMCTA over standard autopsy and histological examination should use water-based contrast, as it has been shown not to alter tissue morphology to ensure accurate comparison between PMCTA and standard histopathology examination. In addition, where PMCTA is necessary to examine for vasculature pathology in post-mortem cases, waterbased contrast can be recommended as it does not affect tissue morphology or immunohistochemical staining in the event a second assessment via conventional method is warranted.

Due to budget and technical limitations at the time, we were unable to compare between imaging and histological findings of the atherosclerotic lesions which could provide answers as to the reliability of PMCTA over conventional autopsy in identifying atherosclerosis. Furthermore, a study comparing the effects of various types of contrast on tissue histology and immunohistochemistry is warranted. Although Ross et al. reported that lipophilic contrast showed extravasation in the intestinal tract while the hydrophilic contrast agent displayed a pronounced soft-tissue enhancement, the number of cases seem too small to fully appreciate advantages and disadvantages of the method ${ }^{24} \cdot{ }^{22}$ Therefore, future studies of larger samples size to compare the effects of other types of contrast media, such as oilbased or air, on the morphology of atherosclerotic plaques and relevant biomarkers of atherosclerosis is warranted to further consider PMCTA as a potential alternative to conventional autopsies.

\section{REFERENCES}

1. Crespo CJ, Di Angelantonio E, Group WCRCW. World Health Organization Cardiovascular Disease Risk Charts: Revised Models To Estimate Risk In 21 Global Regions. The Lancet. 2019;7

2. World Health Organization. Noncommunicable Diseases (NCD) Country Profiles2018. Available from: https://www.who.int/nmh/countries/ mys_en.pdf.

3. Thali MJ, Yen K, Schweitzer W, Vock P, Boesch C, Ozdoba C, et al. Virtopsy, a new imaging horizon in forensic pathology: virtual autopsy by postmortem multislice computed tomography (MSCT) and magnetic resonance imaging (MRI)-a feasibility study. Journal of forensic sciences. 2003;48(2):386-403

4. Rutty GN, Robinson CE, BouHaidar R, Jeffery AJ, Morgan B. The role of mobile computed tomography in mass fatality incidents. Journal of forensic sciences. 2007;52(6):1343-9

5. Hoey BA, Cipolla J, Grossman MD, McQuay N, Shukla PR, Stawicki SP, et al. Postmortem computed tomography, “CATopsy”, predicts cause of death in trauma patients. Journal of Trauma and Acute Care Surgery. 2007;63(5):979 $-86$

6. Leth PM. Computerized tomography used as a routine procedure at postmortem investigations. The American journal of forensic medicine and pathology. 2009;30(3):219-22

7. Dirnhofer R, Jackowski C, Vock P, Potter K, Thali MJ. VIRTOPSY: minimally invasive, imaging-guided virtual autopsy. Radiographics. 2006;26(5):1305-33

8. Rutty G. Are autopsies necessary? Rechtsmedizin. 2007;17(1):21-8 
9. Krantz $\mathrm{P}$, Holtås $\mathrm{S}$. Postmortem computed tomography in a diving fatality. Journal of computer assisted tomography. 1983;7(1):132-4

10. Bisset R, Thomas N, Turnbull I, Lee S. Postmortem examinations using magnetic resonance imaging: four year review of a working service. Bmj. 2002;324(7351):1423-4

11. Donchin Y, Rivkind Al, Bar-Ziv J, Hiss J, Almog J, Drescher M. Utility of postmortem computed tomography in trauma victims. The Journal of trauma. 1994;37(4):552-5; discussion 5-6

12. O'Donnell C, Rotman A, Collett S, Woodford N. Current status of routine post-mortem $\mathrm{CT}$ in Melbourne, Australia. Forensic science, medicine, and pathology. 2007;3(3):226-32

13. Bolliger SA, Thali MJ, Ross S, Buck U, Naether $S$, Vock $P$. Virtual autopsy using imaging: bridging radiologic and forensic sciences. A review of the Virtopsy and similar projects. European radiology. 2008;18(2):273-82

14. Saunders SL, Morgan B, Raj V, Robinson CE, Rutty $\mathrm{GN}$. Targeted post-mortem computed tomography cardiac angiography: proof of concept. International journal of legal medicine. 2011;125(4):609-16

15. Grabherr S, Grimm J, Dominguez A, Vanhaebost $\mathrm{J}$, Mangin $\mathrm{P}$. Advances in post-mortem CTangiography. The British journal of radiology. 2014;87(1036):20130488

16. Rutty G, Smith P, Visser T, Barber J, Amorosa J, Morgan B. The effect on toxicology, biochemistry and immunology investigations by the use of targeted post-mortem computed tomography angiography. Forensic science international. 2013;225(1-3):42-7

17. Palmiere C, Grabherr S, Augsburger M. Postmortem computed tomography angiography, contrast medium administration and toxicological analyses in urine. Legal Medicine. 2015;17(3):157 $-62$

18. Ross SG, Bolliger SA, Ampanozi G, Oesterhelweg L, Thali MJ, Flach PM. Postmortem CT angiography: capabilities and limitations in traumatic and natural causes of death. Radiographics. 2014;34(3):830-46

19. Ni M, Chen WQ, Zhang Y. Animal models and potential mechanisms of plaque destabilisation and disruption. Heart. 2009;95(17):1393-8

20. Getz GS, Reardon CA. Animal models of atherosclerosis. Arteriosclerosis, thrombosis, and vascular biology. 2012;32(5):1104-15

21. Rahman TA, Hassim NF, Zulkafli N, Muid S, Kornain NK, Nawawi H. Atheroprotective effects of pure tocotrienol supplementation in the treatment of rabbits with experimentally induced early and established atherosclerosis. Food \& nutrition research. 2016;60(1):31525

22. Charan J, Kantharia N. How to calculate sample size in animal studies? Journal of pharmacology \& pharmacotherapeutics. 2013;4 (4):303

23. Ross S, Spendlove D, Bolliger S, Christe A, Oesterhelweg L, Grabherr S, et al. Postmortem whole-body CT angiography: evaluation of two contrast media solutions. American Journal of Roentgenology. 2008;190(5):1380-9

24. Palmiere $C$, Binaghi $S$, Doenz F, Bize $P$, Chevallier $C$, Mangin $P$, et al. Detection of hemorrhage source: the diagnostic value of post-mortem CT-angiography. Forensic science international. 2012;222(1-3):33-9

25. Azuma K, Ichimura K, Mita T, Nakayama S, Jin WL, Hirose $T$, et al. Presence of a-smooth muscle actin-positive endothelial cells in the luminal surface of adult aorta. Biochemical and biophysical research communications. 2009;380 (3):620-6

26. Jackowski C, Persson A, Thali MJ. Whole body postmortem angiography with a high viscosity contrast agent solution using poly ethylene glycol as contrast agent dissolver. Journal of forensic sciences. 2008;53(2):465-8

27. Tan C, Liu Y, Li W, Deng F, Liu X, Wang X, et al. Associations of matrix metalloproteinase- 9 and monocyte chemoattractant protein-1 concentrations with carotid atherosclerosis, based on measurements of plaque and intimamedia thickness. Atherosclerosis. 2014;232 (1):199-203

28. Cho A, Reidy MA. Matrix metalloproteinase- 9 is necessary for the regulation of smooth muscle cell replication and migration after arterial injury. Circulation research. 2002;91(9):845-51 